\title{
MIR130A Pre-miRNA
}

National Cancer Institute

\section{Source}

National Cancer Institute. MIR130A Pre-miRNA. NCI Thesaurus. Code C82763.

MIR130A is an oligoribonucleotide that is encoded by the MIR130A gene and is involved in the regulation of gene expression. 